\title{
Mycoplasmas and Human prostate cancer: An exciting but cautionary note
}

\section{Shyh-Ching Lo, Shien Tsai}

A recent study of Barykova, et al. [1] described a significant association between Mycoplasma hominis infection and development of prostate cancer. In the study, the authors detected the presence of $M$. hominis-specific DNA sequences in examining 496 prostate biopsies by PCR obtained from 248 Russian men undergoing cancer diagnosis. The association appeared to be particularly significant in the statistical analysis between $M$. hominis infection and development of the higher grade prostate cancer. A possible role for mycoplasmas in association with human malignancy was first noted during 1960s. Several studies reported isolation of mycoplasmas from human leukemic bone marrow [2-6]. A majority of the mycoplasmas isolated in the studies were identified as Mycoplasma fermentans. However, the mycoplasmaoncogenesis hypothesis failed to advance because the same mycoplasmas later could also be found in nonleukemic children or adults, although the mycoplasmas appeared to be most frequently isolated from patients with leukemia [7]. Koch's postulate of identifying the causative agent in an infectious disease by finding a specific pathogen uniquely present in a particular disease process overshadowed the characteristics in both chronicity and multi-factorial nature of the malignant process.

Mycoplasmas are a heterogeneous group of very small organisms capable of self-replication. The wallfree microbes can cause a wide variety of diseases in animals. Some mycoplasmas cause respiratory or urogenital diseases in humans. However, many other mycoplasmas chronically colonize our respiratory and urogenital tracts without apparent clinical significance [8]. Various mechanisms including "antigenic variation" may be critical in the ability of these microbial species to evade host antibody responses or other elements of immune recognition [9]. Most interestingly, the wallfree mycoplasmas are among the few prokaryotes that can grow essentially "symbiotically" in cultures of mammalian host cells. Mycoplasmas are often growing in a close interaction with mammalian cells for a very long period of time without producing detectable cytopathic effects on the infected host cells. Thus, their infections in cell cultures are commonly unrecognized. However, we believe the interplay between human hosts and these unusual microbes is complex and can be important in the development of various human chronic illnesses including cancers. Our laboratory at the Armed Forces
Institute of Pathology (AFIP) had long been interested in unusual infections of mycoplasmas. We have previously hypothesized chronic infections by the seemingly low virulent microbes could produce a previously unrecognized form of pathogenesis such as neoplasia in the infected mammalian hosts [10].

Over the years, we developed several in vitro mycoplasma-oncogenesis models to demonstrate that infections of mycoplasmas could not only damage DNA or genes of infected mammalian cells but also disturb the cell cycle check points that control normal cell division and the process of apoptosis by providing potent altered cell growth signals [11]. Various signals could continually be sent through close interacting with mycoplasmas from the surface of chronically infected host cells to alter the expression of many different genes, thus significantly affect crucial biological characteristics including control of cell growth $[10,11]$. Thus, we hypothesized that chronic infections by mycoplasmas produced genetic instability and chromosomal aberrations in the mycoplasma-infected cells that however failed to undergo proper apoptosis due to mycoplasma-mediated mitogenic and anti-apoptotic effects would gradually lead to the consequence of malignant transformation [10-12]. Studies of in vitro model systems, which are confined to a fixed condition and may not describe the full range of activities occurring in the infected host, could nevertheless provide valuable information on mechanisms of malignant cell transformation. We believe the chronic persistent infection models of mycoplasmas developed have provided an excellent in vitro model system for cancer research. Most importantly, the in vitro mycoplasma-oncogenesis models developed have a long latency with a multi-stage malignant progression, one of the most common paradigms in naturally occurring human cancers [13].

Mycoplasmas are ubiquitously colonizing in both respiratory and urogenital tracts of human body. The most frequently identified Mycoplasma species in human urogenital tract are $M$. hominis, Ureaplasma urealyticum and M. genitalium. Naturally, one would suspect that some of these urogenital mycoplasmas could also infect or colonize prostate and play an important role in the prostate disease processes like hyperplasia or cancer. In the present publication of Barykova, et al. [1], the results showed a statistically significant association between $M$. hominis infection and development of prostate cancer. 
This study in Russian men is the first report demonstrating the clinical association between $M$. hominis infection in prostate and prostate cancer formation. The significant association could be found in PCR assay of detecting M. hominis-specific DNA, 16S r-RNA and IgG serology studies. The presence of $M$. hominis in some of the prostate biopsies $(6.3 \%)$, although at a lower frequency, could be confirmed by isolation of the mycoplasma by culture. However, the culture isolation and characterization of $M$. hominis from prostate biopsies with or without pathology should be further studied with great care in the future to confirm the association. In a similar note, since many men may have $M$. hominis colonization in the urogenital tract as commensal, the background of anti-M. hominis IgG level was found to be high and significantly varying with men's age. Interestingly, we previously reported men older than 60 showed marked decrease of $M$. hominis infection by serology [14)]. Large-scale epidemiological studies as proposed to be conducted by authors of the article will indeed be required to demonstrate the association between M. hominis infection and prostate cancer contracted mainly by older males. Other urogenital mycoplasmas such as $M$. genitalium should also be further examined for their potential involvement in the prostate disease process. Parallel studies of other human uogenital mycoplasmas could certainly serve as an important control for $M$. hominis.

In addition to large-scale epidemiological studies proposed by Barykova et al. to confirm the significance of their observations, it also would be essential to develop an in vitro cell culture model as well as an animal model for further evaluation of the role of $M$. hominis in the development and progression of prostate carcinogenesis. In this context, Namiki et al. [15] recently reported that chronic exposure of the immortalized human benign prostate cell line, BPH-1 to M. hyorhinis or M. genitalium could induce malignant transformation of the prostate epithelial cells. The prolonged mycoplasma-infected BPH1 cells evidently gained the critical malignant property of forming tumor when introduced into nude mice. We expect that chronic infection with $M$. hominis would also induce BPH-1 cells to undergo malignant transformation, as demonstrated by the infections of $M$. hyorhinis and $M$. genitalium. However, it may be important to note that the immortalized human BPH-1 cell line was previously established by transduction of SV40 T-antigen into primary cultures of prostatic epithelial cells. The BPH-1 cells expressing the viral antigen might be more susceptible to malignant transformation when exposed to the appropriate stimulation such as mycoplasma infections. Therefore, the role of mycoplasma infection played in the BPH-1 cell culture model could be related to the promotion of host cell to undergo further progression to a more malignant phenotype, rather than an initiation of cell transformation (15). Using human PBMCs in the cultures that normally died quickly in the first few weeks, our previous study showed introduction of $M$. fermentans (Mi and PG18 strains) into the PBMC cultures would markedly enhance the blood cells to undergo immortalization [16]. Since all the immortalized lymphocytes obtained after many months were found to be positive for EBV latent membrane protein (LMP1) antigen, the mycoplasma-mediated effect in blood cell immortalization could also be promotional effect, instead of initiation effect.

It has long been documented in both clinical and pathological studies that majority of men older than 50 years have various form/degree of hyperplasia and a very significant proportion $(\sim 50 \%)$ among them already harbor prostate cancer. However, only a small percent of the prostate cancer will continue to progress into highly malignant form and undergo aggressive metastasis. Factor(s) that could be having significant promotional effect in the patients with the progressive malignant disease have never been identified. In this context, although mycoplasma-mediated initiation effect in malignant transformation of mammalian cells has not been well demonstrated, mycoplasma-mediated promotional effect in immortalization and malignant transformation of mammalian cells could be clearly demonstrated in the in vitro models as described above. Chronic and persistent infections with the unusual prokaryotic microbes known to possess potent promotional effect of malignant transformation in vitro could similarly play an important promotional role in the malignant progression of many prostate cancers. Thus, the present finding, if confirmed, would have a highly significant impact and lead to the development of new approaches of preventions, diagnostic tests and therapeutic treatments against prostate cancers.

Table 1: Mycoplasmal infections and development of tumor-like lesions, clinical case reports

\begin{tabular}{lll}
\hline Infections & Tumor-like Lesions & Citations \\
\hline
\end{tabular}

Mycoplasmal pericarditis Left ventricular hemangioma Boden WE et al. [20]

$\begin{array}{ll}\text { M. pneumoniae infection } & \begin{array}{l}\text { Pleural pseudotumor } \\ \text { mesothelial cell proliferation }\end{array}\end{array}$

Abo W et al. [21]

M. pneumoniae infection Pseudotumor of the lung Park SH et al. [22] 
The concept that bacterial infection could lead to cancer development in human was first proposed in the late nineteenth and early twentieth century. The convincing evidence that linked bacterial infection with the development of various cancers was not demonstrated until the association between Helicobacter pylori infection and developments of gastric cancer and mucosa-associated lymphoid tissue (MALT) lymphoma were reported near a hundred years later [17]. Reported evidence of infections by other bacteria species associated with cancer formations in human included Salmonella typhi in gallbladder cancer, Streptococcus bovis in colon cancer, and Chlamydia pneumoniae in lung cancer [18, 19]. Although the present study may be the first demonstration of an association between mycoplasmal infections and cancer development, there were several reports of tumor growth or development of tumor-like lesions in patients with various mycoplasmal infections in lung, pleural sac or pericardium in the past (Table 1). Interestingly, these pseudo-tumors regressed completely following effective antibiotics treatments to eradicate the mycoplasmas. The potent mitogenic effects as well as the potent induction of pro-inflammatory cytokines of the mammalian cells found in the in vitro studies could apparently have equally important in vivo effects in the infected patients. It is not clear if chronic or prolonged mycoplasmal infections could indeed cause genetic alterations and gene instability of these rapidly proliferating cells in these tumor-like lesions, as clearly documented in many in vitro studies of ours and others. Our in vitro model systems have clearly demonstrated once these irreversible genetic events occur the malignant transformation of these cells would become permanent, eradication of mycoplasmas can no longer reverse the continuous/unrestrained growth property of these cells, hence formation of true malignant cancer in the infected patients.

In reviewing the exciting study finding, it is still prudent to note that the high likelihood of both PCR assay and mycoplasma isolation as a result of contamination from various laboratory procedures could often complicate the assessment. This has also been rightly pointed out in another commentary on the current report published in this issue of journal [23]. The study will need to be independently confirmed under a stringent condition. As mentioned earlier, further studies, including solid epidemiological studies and development of animal models, are clearly needed for better assessment of the possible role that, if any, mycoplasmas may be playing in development or progression of prostate cancer. In conclusion, a role for mycoplasmas in any human or animal cancers is still conjectural; however the current report of demonstrating a significant association between $M$. hominis infection and development of prostate cancer has provided an important piece of clinical evidence to support the possibility that chronic infection by the unusual wall-free bacteria could lead to human cancer development. This report should intrigue many more scientists to examine mycoplasma-oncogenesis process and to further investigate potential infectious root in prostate cancer formation or progression.

Tissue Microbiology Laboratory, Division of Cellular and Gene Therapies, Office of Cellular, Tissue and Gene Therapy, Center for Biologics Evaluation and Research, Food and Drug Administration, Bethesda, MD 20892

Email: shyhching.lo@fda.hhs.gov

Received: May 29, 2011;

Published: June 1, 2011;

\section{REFERENCES}

1. Barykova YA, Logunov DU, Shmarov MM, Vinarov AZ, Fiev DN, Vinarova NA, Rakovskaya IV, Baker PS, Shyshynova I, Stephenson AJ, Klein EA, Naroditsky BS, Gintsburg AL, Gudkov AV. Association of Mycoplasma hominis infection with prostate cancer. Oncotarget 2011; 2: 289-297.

2. Barile MF. Mycoplasma and leukemia. Ann N Y Acad Sci. 1967; 143: 557-572.

3. Fallon RJ, Grist NR, Inman DR, Lemcke RM, Negroni G, Woods DA. Further studies of agents isolated from tissue cultures inoculated with human leukemic bone-marrow. $\mathrm{Br}$ Med J. 1965; 2: 388-391.

4. Grace JT Jr, Horoszewicz JS, Stim TB, Mirand EA, James C. Mycoplasmas (PPLO) and human leukemia and lymphoma. Cancer. 1965; 18: 136-1376.

5. Hayflick L, Koprowski H. Direct agar isolation of mycoplasmas from human leukaemic bone marrow. Nature. 1965; 205: 713-714.

6. Murphy WH, Furtado D, Plata E. Possible association between leukemia in children and viruslike agents. JAMA. 1965; 191: 110-115.

7. Murphy WH, Bullis C, Dabich L, Heyn R, Zarafonetis CJ. Isolation of mycoplasma from leukemic and nonleukemic patients. J Natl Cancer Inst. 1970; 45: 243-251.

8. Simecka JW, Davis JK, Davidson MK, Ross SE, Stadtlander CT KH, and Cassell GH. Mycoplasma diseases of animals. In Mycoplasmas: Molecular biology and pathologenesis. J.B. Basement, L.R. Finch, J. Maniloff and R.N. McElhaney, editors. (American Society for Microbiology, Washington, DC.) 1992, pp. 391-416.

9. Leigh SA and Wise KS. Identification and functional mapping of Mycoplasma fermentans P29 adhesin. Infect Immun 2002; 70: 4925-4935.

10. Tsai S, Wear DJ, Shih JW, Lo SC. Mycoplasmas and oncogenesis: persistent infection and multi-stage malignant transformation. Proc Natl Acad Sci U SA. 1995; 92: $10197-$ 10201.

11. Feng SH, Tsai S, Rodriguez J, Lo SC. Mycoplasmal infections prevent apoptosis and induce malignant transformation of interleukin-3-dependent 32D 
hematopoietic cells. Mol Cell Biol. 1999; 19: 7995-8002.

12. Lo SC. Apoptotic, Antiapoptotic, Clastogenic and Oncogenic Effects. In Molecular Biology and Pathogenicity of Mycoplasmas. S. Razin and R. Herrmann (eds) (Kluwer Academic/Plenum Publishers, New York), 2002, pp. 403416.

13. Cavenee WK, Scrable HJ, James CD. Molecular genetics of human cancer predisposition and progression. Mut Res 1991; 247: 199-202.

14. Lo SC, Wang RHY, Grandinetti T, Zou N, Haley CLD, Hayes MM, Wear DJ, Shih JWK. Mycoplasma hominis lipid-associated membrane protein antigens for effective detection of M. hominis-specific antibodies in human. Clinc Infect Dis 2002; 36: 1246-1253.

15. Namiki K, Goodison S, Porvasnik S, Allan S, Iczkowski K, Urbanek C, Reyes L, Sakamoto S, and Rosser C. Persistent exposure to Mycoplasma induces malignant transformation of human prostate cells. PLoS ONE 2009, 4:e6872.

16. Zhang S, Tsai S, Wu TT, Li B, Shih JW, Lo SC. Mycoplasma fermentans infection promotes immortalization of human peripheral blood mononuclear cells in culture. Blood 2004; 104: 4252-4259.

17. Crowe SE. Helicobacter infection, chronic inflammation, and the development of malignancy. Curr Opin Gastroenterol. 2005; 1:32-8.

18. Samaras V, et al. Chronic bacterial and parasitic infections and cancer: a review. J Infect Dev Ctries 2010; 4: 267-281.

19. Chang AH and Parsonnet J. Role of bacteria in oncogenesis. Clin Microbiol Rev. 2010; 23: 837-857.

20. Boden WE, Carleton RA, Khan AH, McEnany MT. Left ventricular hemangioma masquerading as mycoplasma pericarditis. Am Heart J 1983; 106: 771-74.

21. Abo W, Sawada Y, Ogawa S, Toyoguchi A, Chiba S. Mesothelial cell proliferation and localized pleural pseudotumour associated with Mycoplasma pneumoniae infection. Eur J Pediatr 1988; 148: 62-63.

22. Park SH, Choe GY, Kim CW, Chi JG, Sung SH. Inflammatory pseudotumor of the lung in a child with Mycoplasma pneumonia. J Korean Med Sci 1990; 5: 213223.

23. Sfanos KS, Isaacs JT. The "infectious" nature of Human Prostate Cancer: A Cautionary Note. Oncotarget 2011; 2: 281-283 